\title{
Skin dysbiosis in the microbiome in atopic dermatitis is site-specific and involves bacteria, fungus and virus
}

Rie Dybboe Bjerre ${ }^{1 *}$, Jacob Bak Holm², Albert Palleja ${ }^{2}$, Julie Sølberg ${ }^{1}$, Lone Skov ${ }^{3}$ and Jeanne Duus Johansen ${ }^{1}$

\begin{abstract}
Background: Microbial dysbiosis with increased Staphylococcus aureus (S. aureus) colonization on the skin is a hallmark of atopic dermatitis (AD), however most microbiome studies focus on bacteria in the flexures and the microbial composition at other body sites have not been studied systematically.

Objectives: The aim of the study is to characterize the skin microbiome, including bacteria, fungi and virus, at different body sites in relation to $\mathrm{AD}$, lesional state, and $\mathrm{S}$. aureus colonization, and to test whether the nares could be a reservoir for $S$. aureus strain colonization.

Methods: Using shotgun metagenomics we characterized microbial compositions from 14 well defined skin sites from 10 patients with $A D$ and 5 healthy controls.

Results: We found clear differences in microbial composition between AD and controls at multiple skin sites, most pronounced on the flexures and neck. The flexures exhibited lower alpha-diversity and were colonized by S. aureus, accompanied by S. epidermidis in lesions. Malassezia species were absent on the neck in AD. Virus mostly constituted Propionibacterium and Staphylococcus phages, with increased abundance of Propionibacterium phages PHLO41 and PHLO92 and Staphylococcus epidermidis phages CNPH82 and PH15 in AD. In lesional samples, both the genus Staphylococcus and Staphylococcus phages were more abundant. S. aureus abundance was higher across all skin sites except from the feet. In samples where $S$. aureus was highly abundant, lower abundances of $S$. hominis and Cutibacterium acnes were observed. M. osloensis and M. luteus were more abundant in AD. By single nucleotide variant analysis of $S$. aureus we found strains to be subject specific. On skin sites some $S$. aureus strains were similar and some dissimilar to the ones in the nares.
\end{abstract}

Conclusions: Our data indicate a global and site-specific dysbiosis in AD, involving both bacteria, fungus and virus. When defining targeted treatment clinicians should both consider the individual and skin site and future research into potential crosstalk between microbiota in AD yields high potential.

Keywords: Atopic dermatitis, Skin microbiome, Dysbiosis

\footnotetext{
* Correspondence: rie.dybboe.bjerre@regionh.dk

${ }^{1}$ National Allergy Research Centre, Herlev and Gentofte Hospital, University of Copenhagen, Copenhagen, Denmark

Full list of author information is available at the end of the article
}

(C) The Author(s). 2021 Open Access This article is licensed under a Creative Commons Attribution 4.0 International License, which permits use, sharing, adaptation, distribution and reproduction in any medium or format, as long as you give appropriate credit to the original author(s) and the source, provide a link to the Creative Commons licence, and indicate if changes were made. The images or other third party material in this article are included in the article's Creative Commons licence, unless indicated otherwise in a credit line to the material. If material is not included in the article's Creative Commons licence and your intended use is not permitted by statutory regulation or exceeds the permitted use, you will need to obtain permission directly from the copyright holder. To view a copy of this licence, visit http://creativecommons.org/licenses/by/4.0/ The Creative Commons Public Domain Dedication waiver (http://creativecommons.org/publicdomain/zero/1.0/) applies to the data made available in this article, unless otherwise stated in a credit line to the data. 


\section{Background}

The human skin is colonized by a variety of microorganism, interacting with the host and modulating immunity. On healthy human skin, the most abundant bacterial genera are Cutibacterium, Staphylococcus and Corynebacterium with marked topographical diversity [1].

In the common skin disease atopic dermatitis (AD) [2], Staphylococcus aureus expand and conventional culturebased studies [3] find colonizing frequencies of $70 \%$ of lesional and $39 \%$ of non-lesional sites, and $62 \%$ of the nares samples [4]. S. aureus colonization adversely affect disease severity [5]. In recent years, skin microbiomes in AD have been studied in a variety of conditions [5-10]. Most studies are based on sequencing the $16 \mathrm{~S}$ rRNA gene of bacteria. Applying this method, bacterial diversity has been shown to be lower in $\operatorname{AD}[5,7,11]$ and S. epidermidis abundant [5, 12]. Therapy increases diversity [9] and the abundances of Streptococcus, Cutibacterium and Corynebacterium [5]. By applying shotgun sequencing of whole metagenomes a better taxonomical resolution is achieved and all domains can be analysed. Studies applying this method in $\mathrm{AD}$ are emerging $[8,10,13,14]$ and describe specific $S$. aureus strains in severe AD [8], perturbations in the eukaryotic community [13], and define AD subgroups [14].

There is growing evidence of a key role of the microbiome in the pathogenesis of $\mathrm{AD}$ [15]. This is supported by studies showing that microbiome dysbiosis can precede AD in early childhood $[16,17]$. Though there might be a critical window for establishing a healthy microbiome and immune tolerance toward it in early childhood [18], studies applying topical commensals to reestablish a healthy microbiome in AD show improvements in the disease [19-21]. However, benefits of using commensals have been reported to be dependent on skin site, for instance with a treating effect of transplanting $R$. mucosa in the antecubital flexure of AD patients but no effect on hands [19]. In general, most microbiome studies in $\mathrm{AD}$ focus on the body flexures but do not address microbial composition at other body sites. Furthermore, virus in $\mathrm{AD}$ has not been well investigated. Here, we present a case-control study applying shotgun metagenomics to characterize the skin microbiome of $\mathrm{AD}$ patients at different body sites.

\section{Results}

Samples from 5 healthy controls ( 3 women, 2 men), aged 27-63 and 10 patients with AD (7 women and 3 men), aged 24-62 years, were included in this study. Mean Severity Scoring of Atopic Dermatitis (SCORAD) for patients with AD was 30.8 (Table 1). Of 212 samples (including $E$. coli and buffer controls), 91 samples were of insufficient DNA quality and/or amount for sequencing (Table 1). Success of library preparation in lesional samples were $45 \%(32 / 71), 39 \%(27 / 69)$ in non-lesional and $87 \%(61 / 70)$ in controls. Other factors influencing success of library preparation were related to subject and skin site (Table 1).

The sequencing generated an average of 36 million read pairs per sample. Initially, data were described according to the 14 skin sites sampled. When analyzing the effect of lesions, the 14 skin sites were pooled, with a minimum number of 5 samples per group.

\section{Beta diversity revealed characteristic AD skin sites}

Subject explained the majority of the explained microbial variance (PERMANOVA test; $R^{2}=19 \% ; P=0.0001$ ) (Fig. S11), however, the overall skin microbial composition differed significantly between $A D$ and controls (PERMANOVA test; $R^{2}=6 \%, P=0.0001$ ). As visualized on the principal coordinate analysis (PCoA, Fig. 1), samples from the hands and arms, flexures and neck showed the clearest separation according to control or AD (Fig. S1). The lowest separation was observed for perioral and periorbital samples.

\section{Alpha diversity and bacterial species in AD and healthy controls}

Initial exploration of differences in the microbiome composition showed lower bacterial alpha-diversity at the flexures in AD (Fig. S2). The flexures in AD were dominated by the genus Staphylococcus, mostly the species $S$. epidermidis and S. aureus (Fig. 2 and S3).

$S$. aureus was low or undetected in control samples but present at most skin sites among AD patients and occasionally dominated the community (Fig. S3). Individual differences were also seen in $S$. aureus colonization, where AD10 was highly colonized across all skin sites (except from the feet, Fig. S4). Other species more abundant in AD included M. luteus, S. epidermidis, S. saccharolyticus, S. lugdunensis, M. osloensis and Rothia sp. ND6WE1A (Fig. 2 and Table 2). On the contrary species higher in abundance in controls include Cutibacterium acnes, [Propionibacterium] humerusii, Corynebacterium sp. and Corynebacterium singular (Figs. 2, S3 and Table 2).

Feet were dominated by Corynebacterium sp. (Fig. S3). The nares were dominated by $C$. propinquum and Proteobacteria sp., except from those dominated by $S$. aureus in $\mathrm{AD}$ subjects (Fig. 2).

\section{Changes in the mycobiome associated with $A D$}

The bacterial domain dominated the samples of both control and AD. However, fungi were highly present at the neck of controls but not in subjects with AD (Table 2). Malassezia globosa was present in relative abundance ranging from $0.9-2.1 \%$ at antecubital flexures and $0.1-3,4 \%$ at the neck of healthy controls, whereas it 
Table 1 Characterization of the study population and samples

\begin{tabular}{lll}
\hline Characteristics & $\begin{array}{l}\text { Atopic } \\
\text { dermatitis }\end{array}$ & $\begin{array}{l}\text { Healthy } \\
\text { controls }\end{array}$ \\
\hline Subjects analysed, N & 10 & 5 \\
Age, mean (range), years & $47(24-62)$ & 48 (27-63) \\
Female:male ratio & $7: 3$ & $3: 2$ \\
SCORAD & & \\
Mean (range) & $31(20-68)$ & NA \\
Moderate: Severe & $8: 2$ & NA \\
Filaggrin Mutation: Wt: Unknown & $4: 1: 5$ & Unknown \\
HECSI, mean (range) & $10(4-16)$ & NA \\
Treatment & & \\
No & 2 & 5 \\
Steroid & 5 & 0 \\
Systemic & 4 & 0 \\
Nizoral & & 0 \\
Occasionally: likely after study & $1: 1$ & 0 \\
participation & & 0 \\
Co-morbidities & & \\
Asthma & 5 & 0 \\
Hay fever & 5 & \\
CD & 5 & \\
FA & 1 &
\end{tabular}

Skin site successfully sampled, Nonlesional:lesional ratio

$\begin{array}{lll}\text { Nasal } & 8: 1 & 5 \\ \text { Periorbital } & 2: 3 & 5 \\ \text { Perioral } & 4: 4 & 5 \\ \text { Neck } & 2: 3 & 5 \\ \text { Upper inner arms } & 0: 2 & 3 \\ \text { Antecubital fossae } & 1: 3 & 5 \\ \text { Volar forearms } & 0: 2 & 3 \\ \text { Dorsum of hands } & 0: 1 & 3 \\ \text { Palmar hands } & 3: 2 & 3 \\ \text { Between fingers } & 0: 5 & 5 \\ \text { Popliteal flexures } & 1: 1 & 4 \\ \text { Dorsum of feet } & 0: 2 & 5 \\ \text { Arches of feet } & 0: 1 & 5 \\ \text { Between toes } & 6: 2 & 5\end{array}$

The 14 skin areas sampled are listed in the top of the table and in detail include: The neck (the anterior triangle), and bilaterally from the anterior nares, periorbital and perioral areas, antecubital and popliteal flexures (midline $+/-5 \mathrm{~cm}$ ), upper inner arms (starting after the flexural area ending before the armpit, before presence of hair follicles from the armpit), volar forearms (starting after the antecubital fossae to $4 \mathrm{~cm}$ from the wrist), dorsum of the hands and feet (from wrist to joints of the digits), the web spaces between the fingers and toes, palmar hands (from wrist to joints of the digits), and arches of the feet.

Abbreviations: $A D$ atopic dermatitis, $C$ control, $M$ male, $F$ female, SCORAD Severity Scoring Atopic Dermatitis, HECSI Hand Eczema Severity Index, FLG filaggrin gene, UN unknown, $C D$ contact dermatitis, $F A$ food allergy, $W T$ wildtype, Mut mutation, NA not applicable. was almost absent in $\mathrm{AD}$ antecubital flexures $(0-0.3 \%)$ and neck $(0-0.8 \%)$. The same pattern was observed for Malasseziales sp. (Table 2).

\section{Changes in virus associated with $A D$}

The number of viral reads were comparable to fungus and the $E$. Coli control had very few viral reads compared to all the skin samples (Table S2). Both absolute and relative (Fig. S5) abundance of virus (top15) were strongly dependent on the individual. Overall, Propionibacterium (now Cutibacterium) phages and Staphylococcus phages dominated the skin of both healthy controls and $A D$ (Fig. 3). Distinct skin site related patterns appeared with more Propionibacterium phage PHLO41 in the nares and more Staphylococcus phages on feet (Fig. S6). In AD we found increased abundances of Propionibacterium phages, PHLO41 and PHL092, and Staphylococcus epidermidis phages, CNPH82 and PH15 [22] (Fig. 3) - not driven by subject or skin site (Fig. S7B). In lesional skin, Staphylococcus phages expanded (Fig. 3), including phages Ipla5 and Ipla7 (Fig. S7). It is also noteworthy, that the patient AD10 with extensively $S$. aureus colonization also has higher abundances of the Stahylococcus aureus phage phiETA (Fig. S8).

\section{Lesional state and $S$. aureus presence}

We observed control samples grouping together while $\mathrm{AD}$ samples cluster further apart from each other (Fig. 1). Lesional state explained this pattern (Fig. S9A) as lesional sample composition was significantly different from control samples (PERMANOVA test; $R^{2}=7 \%, P=0.0001$ ), again with a large impact of subject on the microbial composition $\left(R^{2}=22 \%, P=0.0001\right)$. However, testing whether the lesional versus non-lesional state explained microbial composition variance did not achieve statistical significance.

In lesional samples, severe AD was associated with higher $S$. aureus colonization $(r=0.63, P=0.00013)$, not seen in non-lesional $(r=0.28, P=0.15)$ (Fig. S9B). $S$. aureus colonization were higher across all skin sites except from the feet in lesional samples (Fig. 4). When S. aureus colonization was high, the relative abundance of S. hominis and C. acnes were lower (Fig. 4).

In the AD flexures, bacterial diversity (Shannon diversity) was lowest at lesional sites and S. epidermidis colonization seemed to accompany $S$. aureus dominance, not however at other sites (Fig. 4).

\section{S. aureus strain colonization}

In total, 42 samples (of 121) had enough S. aureus coverage for single nucleotide variation (SNV) analysis, which were mostly lesional (Fig. S10). In general, the $S$. aureus strains from the same subject exhibited high similarity and lesional samples from three different $\mathrm{AD}$ 


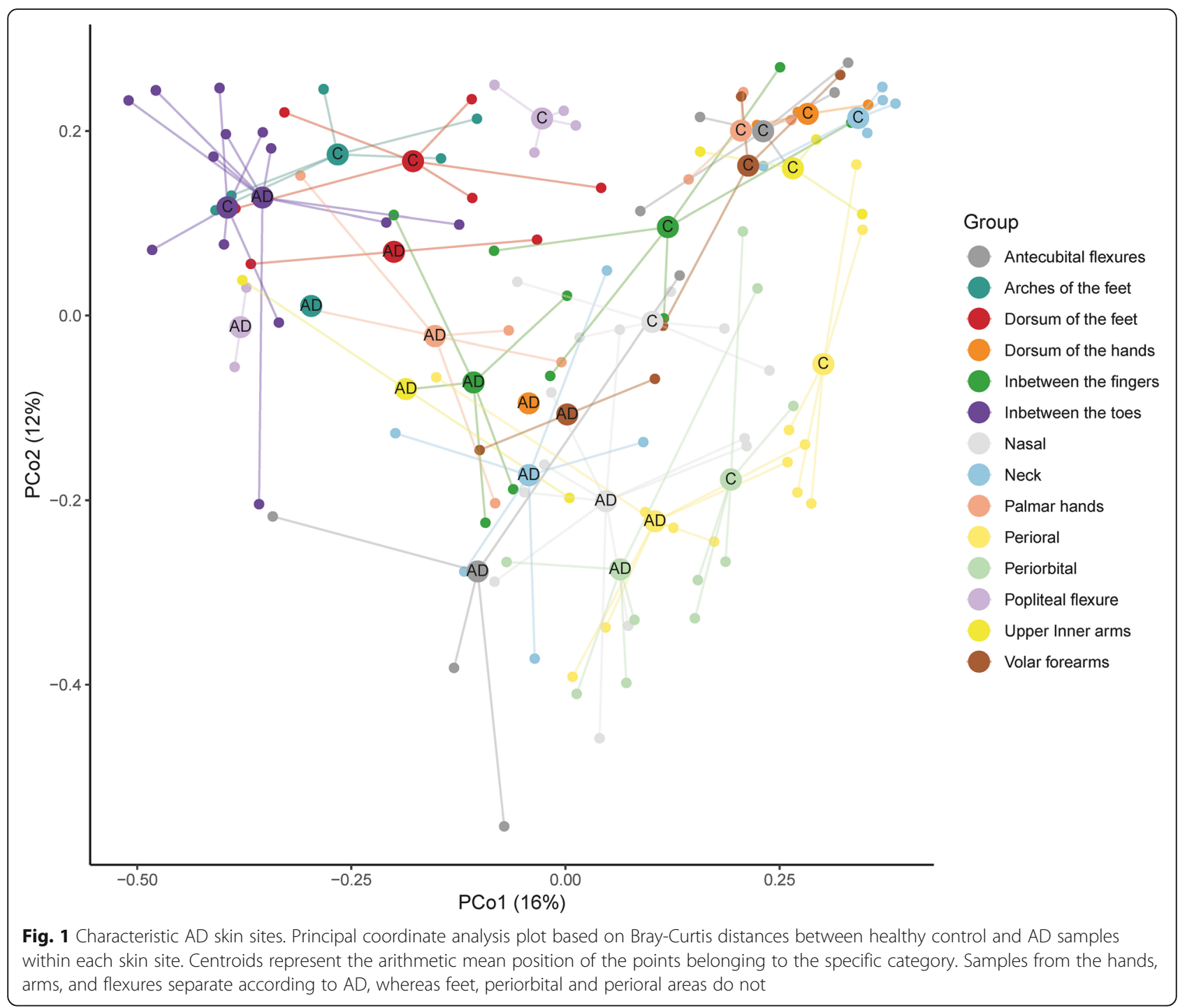

subjects (AD2, 3 and 4) clustered together in the top branch of the tree (Fig. 5A), suggesting that the strains could be lesion and subject-specific and that different $S$. aureus strains may be implied in $\mathrm{AD}$.

In analyzing the nares as reservoir of recurrent $S$. aureus infections, it could be expected that the SNVs from the nares would be similar to the ones in lesional skin, at least at the skin nearby the nose (perioral, periorbital) and at the hands (touching the nares). However, our analysis indicated no such specific pattern (Fig. 5B).

\section{Discussion}

In this study we demonstrated a global skin dysbiosis in $\mathrm{AD}$ at flexures, neck, hands and arms. This is in line with findings from Baurecht and colleagues [23] showing microbial dysbiosis in AD across four skin sites (antecubital flexure, forehead, extensor- and volar forearm). The dysbiosis implicated both bacteria, fungus and virus, and especially our finding of an altered profile of bacteriophages in $\mathrm{AD}$ is intriguing. We also demonstrated some skin sites, feet, periorbital and perioral areas, to have more similar skin microbiome in health and AD.

We found a significantly lower alpha-diversity in AD flexures, and domination by Staphylococcus species, mostly S. aureus and S. epidermidis, as previously reported in the flare condition by Byrd et al. [8] In lesional samples, an increased abundance of Staphylococcus was accompanied by Staphylococcus bacteriophages, including the Staphylococcus epidermidis phages CNPH82 and PH15. As most of these phage's gene content are lysogenic [22], they could insert virulence factors into the bacterial genomes and contribute to a conversion from commensalism to pathogenicity. Likewise, in one patient with severe dermatitis and extensive $S$. aureus colonization, the increased abundance of the phage phiETA could induce more transfer of the virulence gene 


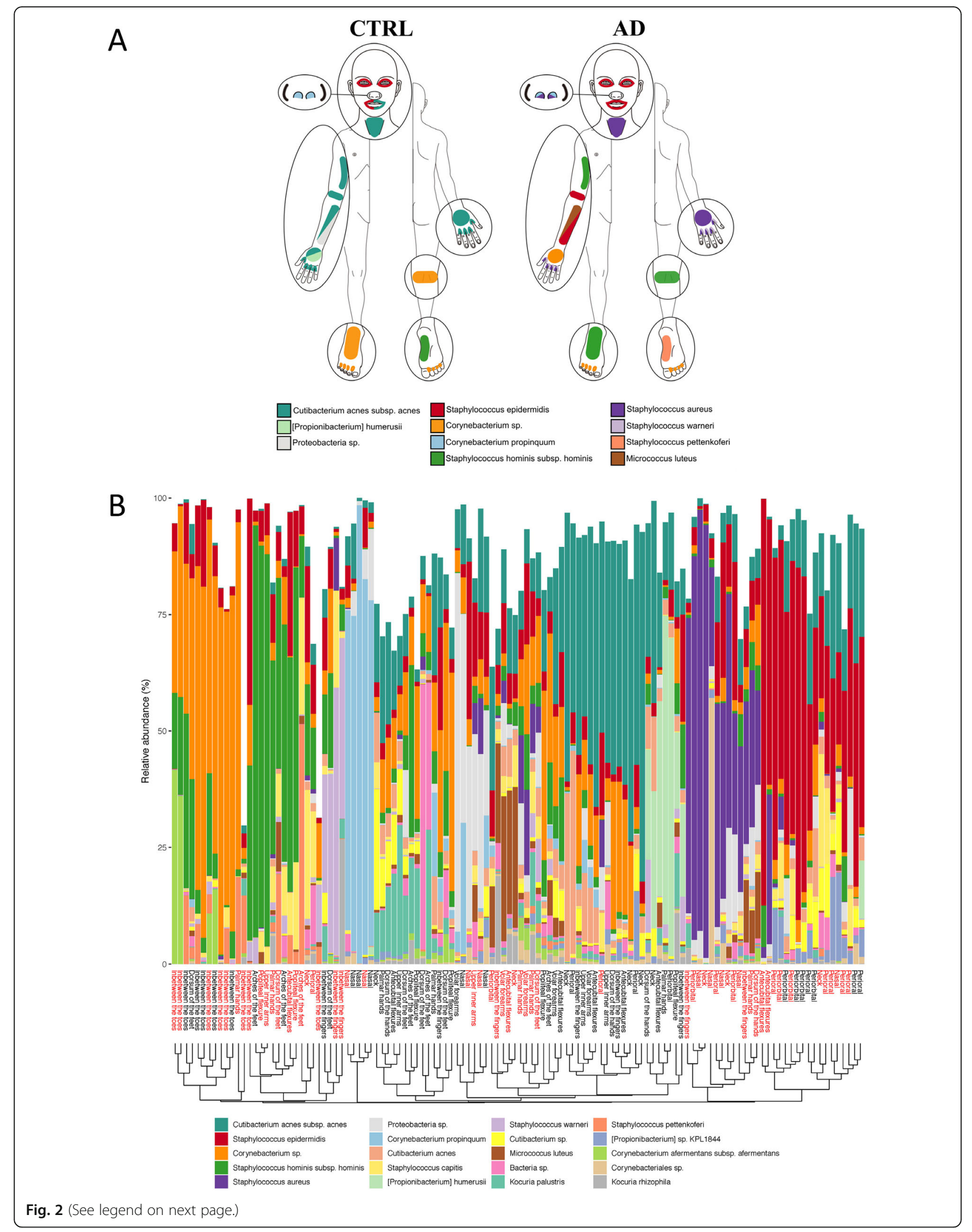


(See figure on previous page.)

Fig. 2 Bacterial species at different skin sites in healthy controls and patients with AD. A, Illustration of the most predominant bacterial species at the 14 non-overlapping skin areas investigated: Two species are depicted at one skin site when the second most abundant specie was within 5\% in total rel. Abundance compared to the most predominant. B. Stacked bar plots of the relative abundances of the 20 bacterial taxa with highest average abundances across all samples, arranged according to similarity (Bray-Curtis). Skin site is stated in red for AD and black for healthy control samples

encoding exfoliative toxin (ET) to S. aureus. This toxin degrades desmosomes in the stratum granulosom [24], whereby the pathogenicity of $S$. aureus would increase and provide a competitive advantage which could lead to increased relative abundance of this bacteria. These hypotheses on potential interactions between bacteria and virus in $\mathrm{AD}$ would be interesting to investigate in future studies.

We also found lower relative abundances of $C$. acnes and [P.] humerusii in AD and a higher colonization of Propionibacterium phages, PHL041 and PHL092. These phages might lyse Cutibacterium ([Propionibacterium]) and result in the lower relative abundance. [P.] humerusii [25] is a common inhabitant of the pilosebaceous unit [26], but to our knowledge this is the first study to report a difference in abundance in control versus skin disease. $C$. acnes has previously been reported to be reduced in AD skin [14, 27, 28]. It is a lipophilic bacteria, and altered sources of fatty acid substrates in AD skin [23, 29] might also restrict its growth. C. acnes ferments glycerol into short-chain fatty acids, including propionic acid, which can inhibit growth of S. aureus [30].

$M$. luteus was more abundant particularly in two $\mathrm{AD}$ subjects and may indicate a certain AD dermotype, as recently suggested [14]. M. luteus has the capability to augment proliferation of virulence of $S$. aureus [31]. The role of $M$. luteus in $\mathrm{AD}$ should be investigated further in future studies. A new important finding of this study is a potential association between $M$. osloensis and $\mathrm{AD}$. Moraxella species are part of the human skin microbiota [32] and M. osloensis is a rare causative organism of human infections [33-37]. It may therefore be relevant to investigate further whether $M$. osloensis is an active player in AD.

No study has yet characterized the skin microbiome of the anterior triangle of the neck in $A D$, which is colonized with high amount of Staphylococcal species, but interestingly, also characterized by a lack of Malassezia species. Malassezia is a genus of lipophilic yeasts and comprises the most common fungi on healthy human skin [38]. The role of Malassezia in AD is debated. It is often attributed a pathogenic role. Especially in a subset of AD patients with symptoms predominating on the head and neck. However, despite that numerous studies have attempted to show a difference in frequency of Malassezia skin colonization in AD patients, there is no such evidence (reviewed by Glatz et al. [38] and Tsakok et al. [39]). As some randomized controlled studies report beneficial effects of anti-fungal treatment [39], we asked the patients whether they have used antifungal treatment (Table 1) and $2 / 5$ might have used Nizoral shampoo around study participation, which could explain some lack of Malassezia in AD, but not in all patients. However, two recent microbiome studies indicate a lack of Malassezia in AD too [40,41] - with one of the studies conducted in an AD prone population, with past AD episodes [40], thus not expected to use antifungal treatment. Poor growth conditions in dry AD skin and absence of $C$. acnes providing substrates for Malassezia could restrict the growth.

Variability in beta-diversity within AD sites are higher than in controls, which we ascribe differences in lesional state. Other endogenous and exogenous factors might also explain larger variability in $A D$ samples. Clinically the disease shows great patient to patient variability, and effort are being put into defining endotypes of the disease [10, 14, 42, 43]. It was recently reported that lesional AD skin is characterized by larger inter- and intra-patient microbiome variability than non-lesional skin [44]. The inter-patient variability mainly originated from $S$. aureus abundance.

Here, lesional samples were characterized by higher $S$. aureus colonization across all skin sites, except from the feet. We find that high abundance of $S$. aureus was accompanied by lower relative abundances of $S$. hominis, which is in line with data from Baurecht et al. showing decreased S. hominis at four AD skin sites [23]. Nakatsuji et al. reports that AD patients lack strains of coagulase-negative Staphylococcus (including $S$. hominis strains) producing antimicrobial peptides against $S$. aureus [21], which can explain their opposing presence in the skin microbiome. In a previous study, reintroducing antimicrobial coagulase negative strains to human subjects with AD decreased $S$. aureus colonization [21]. Other studies have also succeeded in treating $\mathrm{AD}$ with microorganisms $[19,20]$, indicating that microbial transplants could be a promising strategy in AD management and highlighting the clinical relevance of finding skin site-specific species. Our data furthermore indicate that it is 


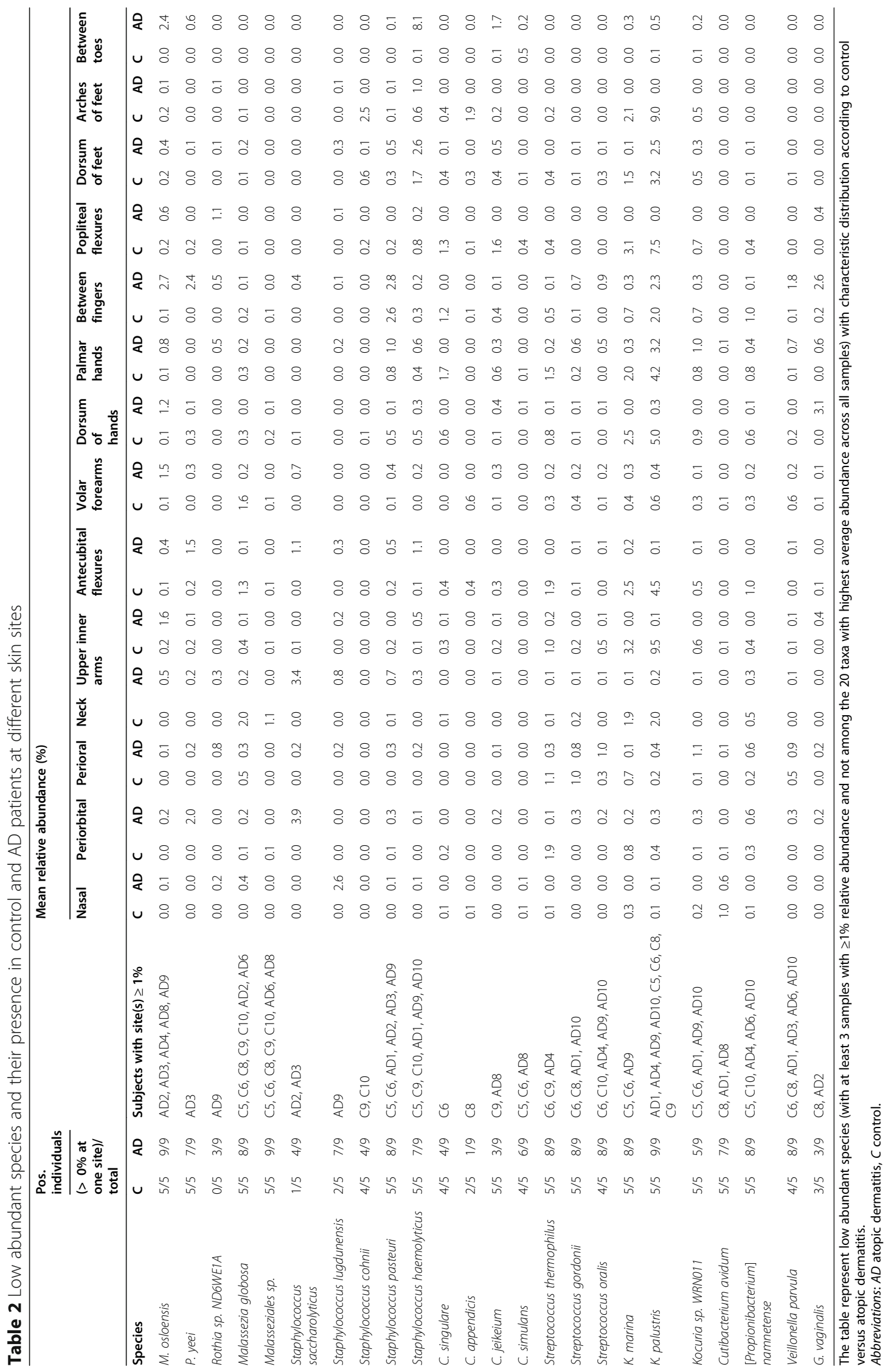




\section{A Top 15 virus species by skin area}

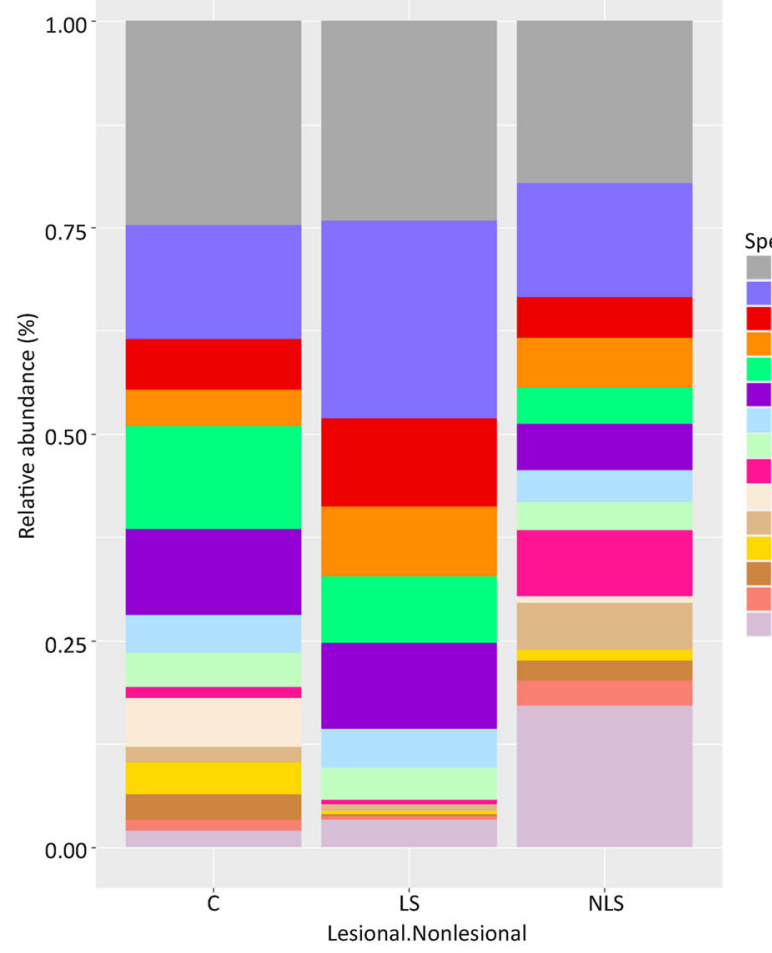

Species

Staphylococcus.phage.StB12 Staphylococcus.phage.IME.SA4

Staphylococcus phage Ipla5

Staphylococcus.phage.StB2

Staphylococcus.phage.StB2O

Staphylococcus.phage.Ipla7

Staphylococcus.phage.CNPH82

Propionibacterium.phage.PHLO41

Propionibacterium phage.PHLO3O

Propionibacterium.phage.PHLO92

Propionibacterium. phage.PHL301

Propionibacterium.phage.PHL141

Propionibacterium. phage.PHL067M10

Escheriachia.virus.Lambda

B

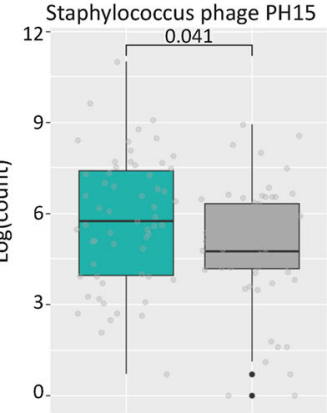

D

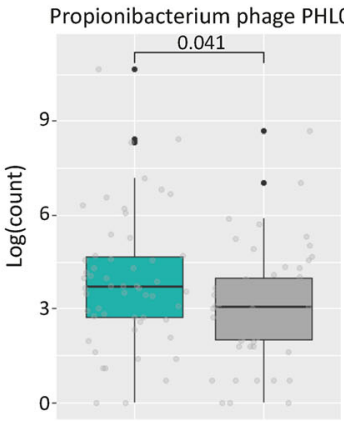

Group 追 $A D$ 官 $C$

C

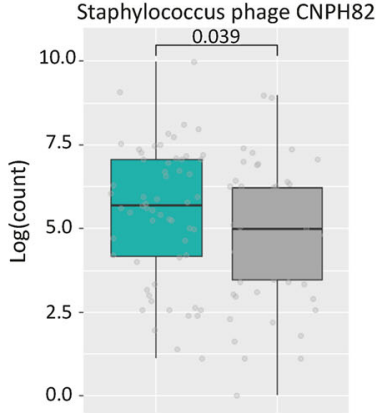

Group 审 $\mathrm{AD}$ 追C

E

Propionibacterium phage PHLO92

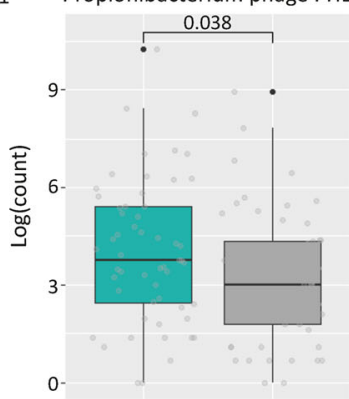

Group 追 $A D$ 审 $C$

Fig. 3 Virus in control and AD. The bar plots represent the relative abundances of the 15 most predominant viruses and show domination of Propionibacterium and Staphylococcal phages. Box plots of the non-normalized read abundances of phages with significant differences between $\mathrm{AD}$ and control 


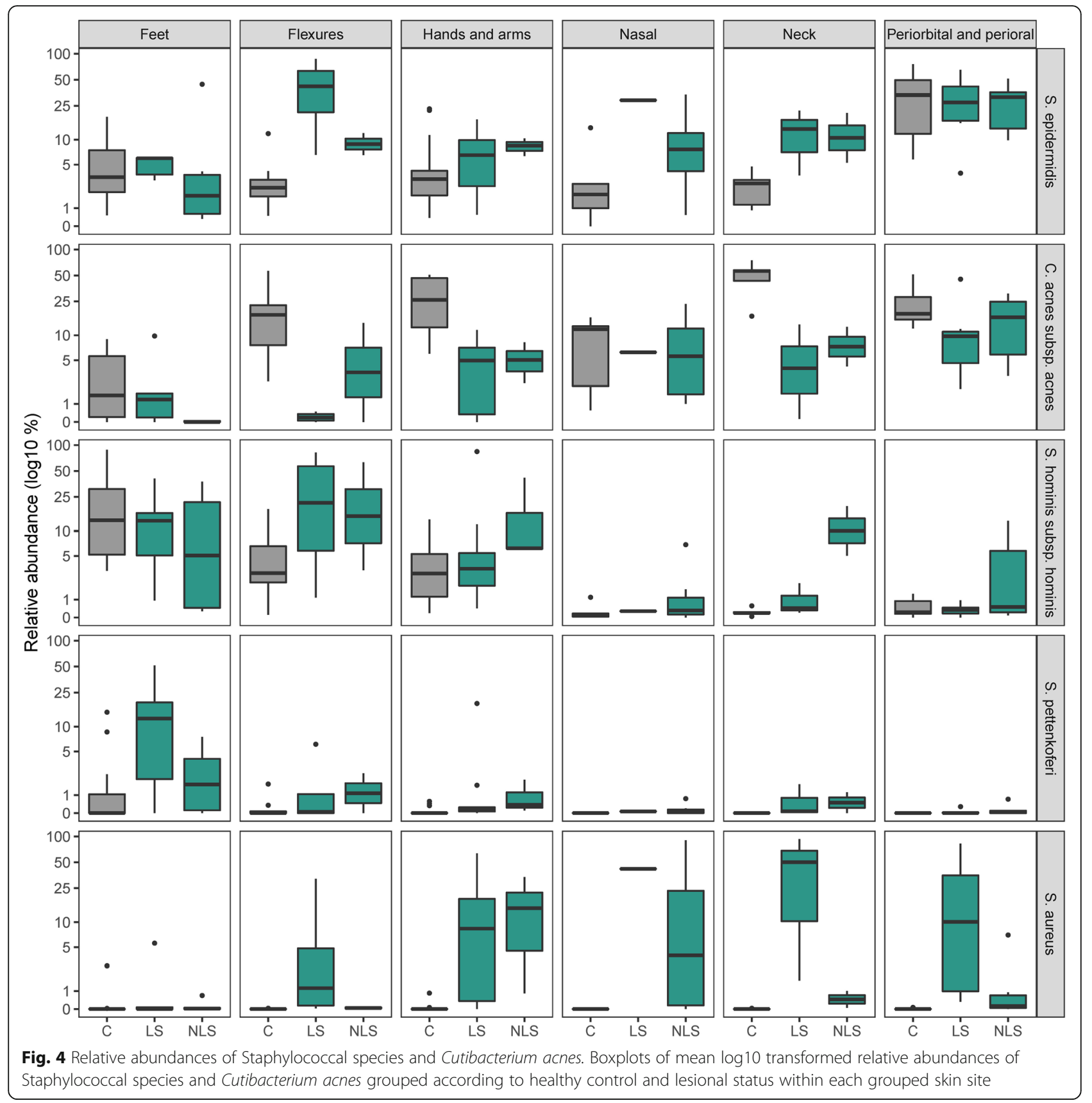

highly relevant to investigate both bacteria, fungi and virus for understanding skin dysbiosis. Using phages for targeting microbial dysbiosis in AD yields potential, which is supported by the specificity of phages [45]. Phage-derived endolysins have been used to target $S$. aureus specifically, however not in AD patients [46].

\section{Strengths and limitations}

Published skin shotgun sequencing data from $\mathrm{AD}$ is sparse and having 121 samples successfully analyzed is a large number. However, a substantial number of samples failed sequencing due to insufficient biomass, making it difficult to evaluate the influence of all relevant factors. The low biomass is a known challenge $[6,47]$. We included AD patients in systemic treatment, which may affect the microbiome. However, even though the patients using topicals were instructed not to apply it $48 \mathrm{~h}$ before, we did not found differences in microbial composition between AD patients in topical versus systemic treatment (PERMANOVA, $R^{2}=4 \%, P=0.98$ ). Another limitation is the use of DNA to characterize skin microbiota as we cannot assess if the microbes are 


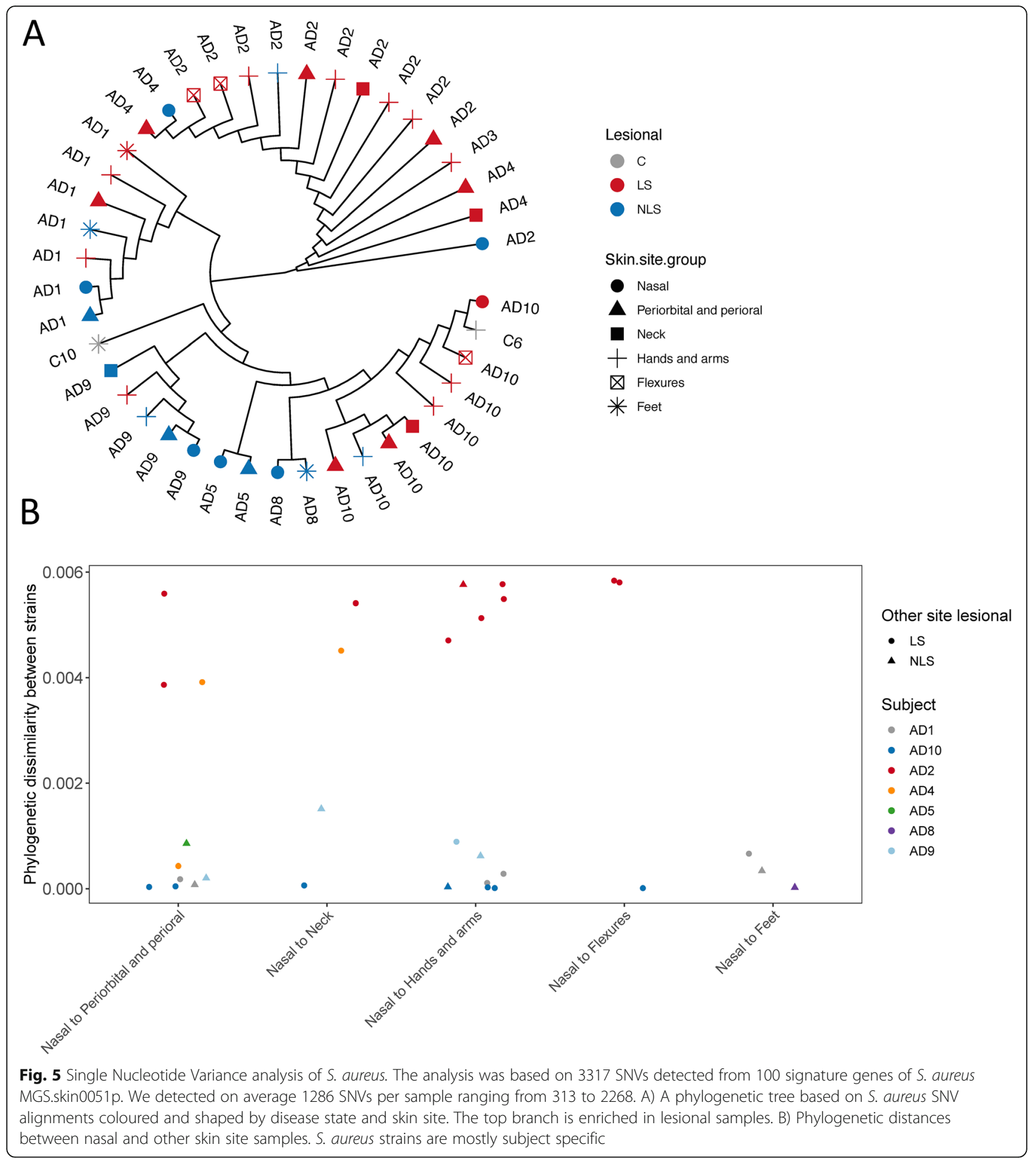

dead or alive or metabolically active. It is also difficult to analyze both bacteria, fungus and virus in the same dataset and it should be underlined that the DNA extraction protocol was optimized for bacteria. It is uncertain if the viral reads come from a phage or phage DNA inserted into a bacterial genome. Reference databases lack annotation for some organisms, which is the case of Malassezia restricta in this study. Studies combining microbiome and transcriptome data in $\mathrm{AD}$ are emerging $[6,10]$ and in general, future studies would benefit from integrating omics data in capturing the flow of information underlying disease states in $\mathrm{AD}$. 


\section{Conclusion}

Though the microbial dysbiosis in AD is global, some sites are more affected than others. In our study, the flexures and neck showed marked taxonomical changes compared to healthy control. The flexures with lower alpha-diversity and high $S$. aureus abundance and high abundance of $S$. epidermidis in lesions, while at the neck Malassezia species were not detected. $S$. aureus colonization was observed across all lesional skin sites except the feet. In general, the $S$. aureus strains were highly similar within subjects both between lesional and non-lesional samples, indicating that more $S$. aureus strains are involved in AD. $S$. aureus may outgrow the coagulase negative $S$. hominis and $C$. acnes. Furthermore, phages targeted [Propionibacterium] and virulent phages such as Staphylococcus phiETA phage might support the growth of S. aureus. $M$. luteus and $M$. osloensis are more abundant in AD and may be active players in the disease.

\section{Methods}

\section{Study participants}

Samples from 10 adult patients with current atopic- and hand dermatitis and 5 healthy age and sex matched controls were enrolled from March to July 2018. All patients were recruited from the Department of Dermatology and Allergy at Herlev and Gentofte Hospital, Denmark. AD had been diagnosed by a physician and confirmed by the UK Working Party Diagnostic Criteria [48] at inclusion. Patients were characterized by demographic data, treatment, co-morbidities, FLG mutations (R501X, 2282del4, and R2447X) when available in their medical records and disease severity assessed by SCORAD and Hand Eczema Severity Index (HECSI) (Table 1). Exclusion criteria included active infections, use of antibiotics or probiotics within the past 4 weeks and for healthy volunteers a history of eczema. Two days before sampling, subjects were instructed not to shower or use topicals.

\section{Sampling, DNA extraction and sequencing}

Skin samples were collected using eSwabs from nonoverlapping areas on 14 sites (Table 1) as described previously [49]. When eczema was present, the area affected, and morphology were described (Table S1).

DNA was extracted using QIAamp DNA Microbiome Kit (QIAGEN, lot no.: 154026306) according to manufactures' protocol. The DNA was randomly sheared into fragments of around 350 basepairs by sonication. Library preparation was performed with the NEBNext Ultra II Library Prep Kit for Illumina (New England Biolabs). Paired-end sequencing $(2 \times 150$ basepair $)$ was performed on an Illumina platform.
Preprocessing of sequencing data and mapping reads to the gene catalog

For analyses of the bacteriome and mycobiome, adaptor removal from raw FASTQ files was performed using KneadData (v. 0.6.1) and Trimmomatic. Trimmed reads shorter than 100 bases were discarded. PCR/optical duplicates were removed using samtools (v. 1.6). Host reads mapping to the human reference genome GRCh38 (with Bowtie2 v. 0.0.3.2) were excluded. Read pairs in which both reads passed filtering were retained and mapped using BWA mem (v 0.7.16a) to a reference gene catalogue built by Clinical Microbiomics from shotgun sequencing data from 1972 skin microbiome samples, containing 4.4 million non-redundant genes and 234 skin-associated metagenomic species (MGS, v3.0) [50] with highly coherent gene abundance and base composition have been identified (as described in Nielsen et al.).

To taxonomically annotate the MGSs, all the catalog genes has been blasted to the NCBI RefSeq genome database (2019-02-18). A MGS was considered detected if read pairs were mapped to at least three of its $100 \mathrm{sig}$ nature genes. Normalization was done to the effective gene length and then to sum $100 \%$, resulting in a relative abundance estimate of each MGS.

For analyses of virus, quality processed FASTQ files (AdaptorRemoval-2.1.3) were assigned taxonomic labels using Kraken 2.

\section{Ultrahigh-resolution phylogenetic profiling}

For all samples in which S. aureus (MGS.skin0051p) was detected, we extracted the reads aligning to 100 signature genes of MGS.skin0051p and used the BCFtools (v. 1.6) multiallelic genotype caller to summarize the counts of each base observed in each position (requiring: sequencing depth $\geq 5$ and $\geq 80 \%$ major allele fraction and filtering to remove indels and SNVs near indels). Samples with at least $40 \%$ of the positions with a called base were retained for further analysis.

Maximum-Likelihood phylogenetic trees with pairwise distances were inferred using IQ-TREE (v. 1.6) based on the alignment of the Single Nucleotide Variants (SNV) considered from the 100 signature genes for MGS.skin0051p. By using ModelFinder Plus we selected the best substitution model estimated separately for each gene. This resulted in phylogenetic trees where each branch represents the most dominant $S$. aureus strain in a given sample. The phylogenetic distances matrix was constructed from all pairwise tree-branch length distances between any two samples in the tree (i.e. patristic pairwise distances).

\section{Statistical analyses}

Descriptive data on relative abundances was both analysed according to individuals and disease groups. Beta-diversity 
was estimated by Bray-Curtis dissimilarity among samples and alpha-diversity using Shannon's index, both measures were based on MGS abundances. Permutational multivariate analysis of variance (PERMANOVA) was used to assess the effects of disease (AD vs Control) or lesional state (Control, Lesional and Non-lesional), considering a nested model of disease within skin area and adjusting for subject variability. Pearson correlations were calculated between $\mathrm{AD}$ severity scores and $S$. aureus abundance. Wilcoxon signed-rank test was used to compare viral abundances between two groups. Outliers in box plots were defined by the interquartile range rule. Visualizations and statistics were conducted in $\mathrm{R}$ ( $\mathrm{R}$ core team, version 4.0.4, http:// www.R-project.org/), where we also used the application Pavian for gathering Kraken reports.

\section{Abbreviations}

AD: Atopic dermatitis; S. aureus: Staphylococcus aureus; S. epidermidis: Staphylococcus epidermidis; S. hominis: Staphylococcus hominis; SCORAD: Severity Scoring of Atopic Dermatitis; PERMANOVA: Permutational multivariate analysis of variance; PCoA: Principal coordinate analysis; $E$. coli: Escherichia coli; S. saccharolyticus: Staphylococcus saccharolyticus; S. lugdunensis: Staphylococcus lugdunensis; C. acnes: Cutibacterium acnes; SNV: Single nucleotide variation; [P.] humerusii: [Propionibacterium] humerusii; M. Iuteus: Micrococcus luteus; M. osloensis: Moraxella osloensis.

\section{Supplementary Information}

The online version contains supplementary material available at https://doi. org/10.1186/s12866-021-02302-2

Additional file 1.

\section{Acknowledgements}

We thank Anna Bünning Olsson for illustrating the skin dysbiosis in Fig. 2A.

\section{Authors' contributions}

RDB, JDJ and LS initiated the study. LS and RDB included subjects, sampled and did clinical scorings. RDB extracted DNA. JBH and AP sequenced the samples and performed analyses of the bacteriome and mycobiome. JS and $\mathrm{RDB}$ analysed the virus. RDB wrote the main manuscript. All authors read and approved the final manuscript.

\section{Funding}

A grant from Danish Environmental Protection Agency is supporting salary of the scientists at the National Allergy Research Centre. Running costs were supported by Aage Bang's Foundation, A.P. Møller Foundation and Inge and Asker Larsen's Foundation.

\section{Availability of data and materials}

The sequencing datasets generated and analysed during the study are not publicly available due to privacy of the participants but are available from the corresponding author upon reasonable request.

\section{Declarations}

\section{Ethics approval and consent to participate}

This study was approved by the ethics committee of the Capital Region of Denmark (H-16020971) and all participants gave informed consent. All methods were carried out in accordance with relevant guidelines and regulations.

\section{Consent for publication}

Consent for publication has been obtained from all study participants.

\section{Competing interests}

None.

\section{Author details}

${ }^{1}$ National Allergy Research Centre, Herlev and Gentofte Hospital, University of Copenhagen, Copenhagen, Denmark. ${ }^{2}$ Clinical Microbiomics, Fruebjergvej 3, 2100 Copenhagen, Denmark. ${ }^{3}$ Department of Dermatology and Allergy, Herlev and Gentofte Hospital, University of Copenhagen, Copenhagen, Denmark.

Received: 8 April 2021 Accepted: 28 August 2021

Published online: 23 September 2021

\section{References}

1. Grice EA, Kong HH, Conlan S, Deming CB, Davis J, Young AC, et al. Topographical and temporal diversity of the human skin microbiome. Science. 2009:324(5931):1190-2. https://doi.org/10.1126/science.1171700.

2. Deckers IA, et al. Investigating international time trends in the incidence and prevalence of atopic eczema 1990-2010: a systematic review of epidemiological studies. PLoS One. 2012;7(7):e39803. https://doi.org/10.13 71/journal.pone.0039803.

3. Leyden JJ, Marples RR, Kligman AM. Staphylococcus aureus in the lesions of atopic dermatitis. Br J Dermatol. 1974;90(5):525-30. https://doi.org/10.1111/ j.1365-2133.1974.tb06447.x.

4. Totte JE, et al. Prevalence and odds of Staphylococcus aureus carriage in atopic dermatitis: a systematic review and meta-analysis. Br J Dermatol. 2016;175(4):687-95. https://doi.org/10.1111/bjd.14566.

5. Kong HDH, et al. Temporal shifts in the skin microbiome associated with disease flares and treatment in children with atopic dermatitis. Genome Res. 2012;22(5):850-9. https://doi.org/10.1101/gr.131029.111.

6. Altunbulakli C, Reiger M, Neumann AU, Garzorz-Stark N, Fleming M, Huelpuesch C, et al. Relations between epidermal barrier dysregulation and Staphylococcus species-dominated microbiome dysbiosis in patients with atopic dermatitis. J Allergy Clin Immunol. 2018;142(5):1643-1647.e12. https://doi.org/10.1016/j.jaci.2018.07.005.

7. Clausen ML, Agner T, Lilje B, Edslev SM, Johannesen TB, Andersen PS. Association of Disease Severity with Skin Microbiome and Filaggrin Gene Mutations in adult atopic dermatitis. JAMA Dermatol. 2018;154(3):293-300. https://doi.org/10.1001/jamadermatol.2017.5440.

8. Byrd AL, et al. Staphylococcus aureus and Staphylococcus epidermidis strain diversity underlying pediatric atopic dermatitis. Sci Transl Med. 2017;9. https://doi.org/10.1126/scitransImed.aal4651.

9. Callewaert C, et al. IL-4Ralpha Blockade by Dupilumab Decreases Staphylococcus aureus Colonization and Increases Microbial Diversity in Atopic Dermatitis. J Invest Dermatol. 2020;140:191-202.e197. https://doi. org/10.1016/j.jid.2019.05.024.

10. Leung DYM, et al. The nonlesional skin surface distinguishes atopic dermatitis with food allergy as a unique endotype. Sci Transl Med. 2019;11. https://doi.org/10.1126/scitrans/med.aav2685.

11. Song $L$, et al. Cheek Microbial Communities Vary in Young Children with Atopic Dermatitis in China. Dermatology. 2019:1-10. https://doi.org/10.1159/ 000502469.

12. Totte JEE, et al. Nasal and skin microbiomes are associated with disease severity in paediatric atopic dermatitis. Br J Dermatol. 2019;181(4):796-804 https://doi.org/10.1111/bjd.17755.

13. Chng KR, et al. Whole-metagenome profiling reveals distinct population and functional differences in skin microbiomes of individuals predisposed to atopic dermatitis. Br J Dermatol. 2016;174:e49.

14. Tay ASL, Li C, Nandi T, Chng KR, Andiappan AK, Mettu VS, et al. Atopic dermatitis microbiomes stratify into ecological dermotypes enabling microbial virulence and disease severity. J Allergy Clin Immunol. 2020;147(4): 1329-40. https://doi.org/10.1016/j.jaci.2020.09.031.

15. Bjerre RD, Bandier J, Skov L, Engstrand L, Johansen JD. The role of the skin microbiome in atopic dermatitis: a systematic review. Br J Dermatol. 2017; 177(5):1272-8. https://doi.org/10.1111/bjd.15390.

16. Meylan P, Lang C, Mermoud S, Johannsen A, Norrenberg S, Hohl D, et al. Skin colonization by Staphylococcus aureus precedes the clinical diagnosis of atopic dermatitis in infancy. J Invest Dermatol. 2017;137(12):2497-504. https://doi.org/10.1016/j.jid.2017.07.834. 
17. Kennedy EA, Gallagher A, Hourihane JO, Fallon P, Murray D, Segre JA, et al. Skin microbiome alterations in infants before clinical atopic dermatitis. J Invest Dermatol. 2016;136(5):S84. https://doi.org/10.1016/j.jid.2016.02.512.

18. Leech JM, et al. Toxin-Triggered Interleukin-1 Receptor Signaling Enables EarlyLife Discrimination of Pathogenic versus Commensal Skin Bacteria. Cell Host Microbe. 2019:26:795-809.e795. https://doi.org/10.1016/j.chom.2019.10.007.

19. Myles $\mid$ A, et al. First-in-human topical microbiome transplantation with Roseomonas mucosa for atopic dermatitis. JCl Insight. 2018;3. https://doi. org/10.1172/jci.insight.120608.

20. Gueniche A, Knaudt B, Schuck E, Volz T, Bastien P, Martin R, et al. Effects of nonpathogenic gram-negative bacterium Vitreoscilla filiformis lysate on atopic dermatitis: a prospective, randomized, double-blind, placebocontrolled clinical study. Br J Dermatol. 2008;159(6):1357-63. https://doi. org/10.1111/j.1365-2133.2008.08836.x.

21. Nakatsuji T, et al. Antimicrobials from human skin commensal bacteria protect against Staphylococcus aureus and are deficient in atopic dermatitis. Sci Transl Med. 2017;9. https://doi.org/10.1126/scitranslmed.aah4680.

22. Daniel A, Bonnen PE, Fischetti VA. First complete genome sequence of two Staphylococcus epidermidis bacteriophages. J Bacteriol. 2007;189(5):2086-100. https://doi.org/10.1128/jb.01637-06.

23. Baurecht $\mathrm{H}$, et al. Epidermal lipid composition, barrier integrity, and eczematous inflammation are associated with skin microbiome configuration. J Allergy Clin Immunol. 2018;141:1668-1676.e1616. https:// doi.org/10.1016/j.jaci.2018.01.019.

24. Yamaguchi T, Hayashi T, Takami H, Nakasone K, Ohnishi M, Nakayama K, et al. Phage conversion of exfoliative toxin a production in Staphylococcus aureus. Mol Microbiol. 2000;38(4):694-705. https://doi.org/10.1046/j.1365-2 958.2000.02169.x.

25. Butler-Wu SM, Sengupta DJ, Kittichotirat W, Matsen FA 3rd, Bumgarner RE. Genome sequence of a novel species, Propionibacterium humerusii. J Bacteriol. 2011;193(14):3678. https://doi.org/10.1128/jb.05036-11.

26. Fitz-Gibbon S, Tomida S, Chiu BH, Nguyen L, du C, Liu M, et al. Propionibacterium acnes strain populations in the human skin microbiome associated with acne. J Invest Dermatol. 2013;133(9):2152-60. https://doi. org/10.1038/jid.2013.21.

27. Dekio I, Sakamoto M, Hayashi H, Amagai M, Suematsu M, Benno Y. Characterization of skin microbiota in patients with atopic dermatitis and in normal subjects using 16S rRNA gene-based comprehensive analysis. J Med Microbiol. 2007;56(12):1675-83. https://doi.org/10.1099/jmm.0.47268-0.

28. Fyhrquist N, Muirhead G, Prast-Nielsen S, Jeanmougin M, Olah P, Skoog T, et al. Microbe-host interplay in atopic dermatitis and psoriasis. Nat Commun. 2019;10(1):4703. https://doi.org/10.1038/s41467-019-12253-y.

29. van Smeden J, Bouwstra JA. Stratum Corneum lipids: their role for the skin barrier function in healthy subjects and atopic dermatitis patients. Curr Probl Dermatol. 2016;49:8-26. https://doi.org/10.1159/000441540.

30. Wang Y, Dai A, Huang S, Kuo S, Shu M, Tapia CP, et al. Propionic acid and its esterified derivative suppress the growth of methicillin-resistant Staphylococcus aureus USA300. Benefic Microbes. 2014;5(2):161-8. https://doi.org/10.3920/bm2013.0031.

31. Boldock E, Surewaard BGJ, Shamarina D, Na M, Fei Y, Ali A, et al. Human skin commensals augment Staphylococcus aureus pathogenesis. Nat Microbiol. 2018;3(8):881-90. https://doi.org/10.1038/s41564-018-0198-3.

32. Lim JY, Hwang I, Ganzorig M, Huang SL, Cho GS, Franz CMAP, et al. Complete genome sequences of three Moraxella osloensis strains isolated from human skin. Genome Announc. 2018;6(3):e01509-17. https://doi.org/1 0.1128/genomeA.01509-17.

33. Sifri CD, Brassinga AKC, Flohr T, Kinchen JM, Hazen KC, Sawyer RG, et al. Moraxella osloensis bacteremia in a kidney transplant recipient. Transpl Int. 2008;21(10):1011-3. https://doi.org/10.1111/j.1432-2277.2008.00727.x.

34. Roh KH, Kim CK, Koh E, Kim MS, Yong D, Park SC, et al. Three cases of Moraxella osloensis meningitis: a difficult experience in species identification and determination of clinical significance. J Korean Med Sci. 2010;25(3):501-4. https://doi.org/10.3346/jkms.2010.25.3.501.

35. Alkhatib NJ, Younis MH, Alobaidi AS, Shaath NM. An unusual osteomyelitis caused by Moraxella osloensis: a case report. Int J Surg Case Rep. 2017;41: 146-9. https://doi.org/10.1016/j.ijscr.2017.10.022.

36. Han XY, Tarrand JJ. Moraxella osloensis blood and catheter infections during anticancer chemotherapy: clinical and microbiologic studies of 10 cases. Am J Clin Pathol. 2004;121(4):581-7. https://doi.org/10.1309/qbb3-avcm-gwa3-k1xk.

37. Buchman AL, Pickett MJ, Mann L, Ament ME. Central venous catheter infection caused by Moraxella osloensis in a patient receiving home parenteral nutrition. Diagn Microbiol Infect Dis. 1993;17(2):163-6. https://doi.org/10.1016/0732-8893(93)90028-6.

38. Glatz M, Bosshard PP, Hoetzenecker W, Schmid-Grendelmeier P. The role of malassezia spp in atopic dermatitis. J Clin Med. 2015;4(6):1217-28. https://doi.org/10.3390/jcm4061217.

39. Tsakok T, Schulenburg H, Smith C, Till S, Flohr C. The role of yeast in atopic dermatitis revisited: a critical appraisal. Curr Dermatol Rep. 2015;4(4):228-40. https://doi.org/10.1007/s13671-015-0123-4.

40. Chng KR, Tay ASL, Li C, Ng AHQ, Wang J, Suri BK, et al. Whole metagenome profiling reveals skin microbiome-dependent susceptibility to atopic dermatitis flare. Nat Microbiol. 2016;1(9):16106. https://doi.org/10.1038/ nmicrobiol.2016.106

41. Moosbrugger-Martinz V, Hackl H, Gruber R, Pilecky M, Knabl L, Orth-Höller D, et al. Initial evidence of distinguishable bacterial and fungal Dysbiosis in the skin of patients with atopic dermatitis or Netherton syndrome. J Invest Dermatol. 2021;141(1):114-23. https://doi.org/10.1016/j.jid.2020.05.102.

42. Paternoster L, Savenije OEM, Heron J, Evans DM, Vonk JM, Brunekreef B, et al. Identification of atopic dermatitis subgroups in children from 2 longitudinal birth cohorts. J Allergy Clin Immunol. 2018;141(3):964-71. https://doi.org/10.1016/j.jaci.2017.09.044

43. Dyjack N, Goleva E, Rios C, Kim BE, Bin L, Taylor P, et al. Minimally invasive skin tape strip RNA sequencing identifies novel characteristics of the type 2high atopic dermatitis disease endotype. J Allergy Clin Immunol. 2018; 141(4):1298-309. https://doi.org/10.1016/j.jaci.2017.10.046.

44. van den Munckhof EHA, et al. Inter- and Intra-patient Variability Over Time of Lesional Skin Microbiota in Adult Patients with Atopic Dermatitis. Acta Derm Venereol. 2020;100:adv00018. https://doi.org/10.2340/00015555-3373.

45. Shimamori Y, et al. Staphylococcal Phage in Combination with Staphylococcus Epidermidis as a Potential Treatment for Staphylococcus Aureus-Associated Atopic Dermatitis and Suppressor of Phage-Resistant Mutants. Viruses. 2020;13. https://doi.org/10.3390/v13010007.

46. Tham EH, Koh E, Common JEA, Hwang IY. Biotherapeutic approaches in atopic dermatitis. Biotechnol J. 2020;15(10):e1900322. https://doi.org/10.1 002/biot.201900322.

47. Hannigan GD, Hodkinson BP, McGinnis K, Tyldsley AS, Anari JB, Horan AD, et al. Culture-independent pilot study of microbiota colonizing open fractures and association with severity, mechanism, location, and complication from presentation to early outpatient follow-up. J Orthop Res. 2014;32(4):597-605. https://doi.org/10.1002/jor.22578.

48. Williams HC, et al. The U.K. working Party's diagnostic criteria for atopic dermatitis. I. Derivation of a minimum set of discriminators for atopic dermatitis. Br J Dermatol. 1994;131(3):383-96. https://doi.org/10.1111/j.13 65-2133.1994.tb08530.x.

49. Bjerre RD, Hugerth LW, Boulund F, Seifert M, Johansen JD, Engstrand L. Effects of sampling strategy and DNA extraction on human skin microbiome investigations. Sci Rep. 2019;9(1):17287. https://doi.org/10.1038/ s41598-019-53599-z.

50. Nielsen $\mathrm{HB}$, et al. Identification and assembly of genomes and genetic elements in complex metagenomic samples without using reference genomes. Nat Biotechnol. 2014;32(8):822-8. https://doi.org/10.1038/nbt.2939.

\section{Publisher's Note}

Springer Nature remains neutral with regard to jurisdictional claims in published maps and institutional affiliations.

Ready to submit your research? Choose BMC and benefit from:

- fast, convenient online submission

- thorough peer review by experienced researchers in your field

- rapid publication on acceptance

- support for research data, including large and complex data types

- gold Open Access which fosters wider collaboration and increased citations

- maximum visibility for your research: over $100 \mathrm{M}$ website views per year

At $\mathrm{BMC}$, research is always in progress.

Learn more biomedcentral.com/submissions 